Trauma Berufskrankh $2005 \cdot 7$ [Suppl 1]:S33-S38 DOI 10.1007/s10039-004-0941-2

Online publiziert: 5. November 2004

(c) Springer Medizin Verlag 2004

M. Faschingbauer - O. Pingen · C. Jürgens · D. Wolter

Berufsgenossenschaftliches Unfallkrankenhaus Hamburg

\title{
Erfahrungen mit winkelstabilen Plattensystemen bei periprothetischen Frakturen
}

dung zwischen Schraubenkopf und Platte, den so genannten Fixateur-interne-Systemen, werden die Lasten gleichmäßig flächenhaft über der gesamten Schraubenlänge auf den Knochen übertragen. Die Möglichkeit, Schrauben in frei wählbare Richtung zu platzieren, ist dabei aus mehreren Gründen unverzichtbar:

- Durch eine schrägverlaufende Schraubenlage kann eine lange und große Schraubenkontaktfläche erreicht werden. Dies bedeutet eine weitere Verbesserung der Lastübertragung.

- Zum anderen erlaubt die freie Wählbarkeit der Richtung, die Schraube in die mechanisch beste Position zu bringen.

- Drittens kann der Operateur die Schraubenlage der individuellen Situation anpassen.

\section{Material und Methode}

Zur Versorgung von periprothetischen Frakturen wird der Druckplattenfixateur interne und das TiFix-System eingesetzt.

\section{Fixateur-interne-Systeme}

Beim Druckplattenfixateur handelt es sich um eine winkelstabile Wellenplatte aus Titan. Nach Fixierung der Platte mittels Schrauben wird auf diese Platte eine Deckelplatte geschraubt. Dadurch werden die Schraubenköpfe in der Platte in der jeweiligen Richtung fixiert.

Als weiteres Fixateur-interne-System wird der Kondylenfixateur (TiFix) ein- gesetzt. Hierbei ist die Platte aus weicherem Titan (Titan o). In diese Platte wird mit einem Gewindedränger ein Gewinde geformt. Die Schraube, welche aus härterem Titan (Titan 4) besteht, trägt an ihrem Kopf ein Gewinde. Durch Einschrauben geht das Gewinde im Schraubenkopf mit dem gedrängten Gewinde im Plattenloch eine winkelstabile Verbindung in der gewünschten Richtung ein. Das Gewinde des Schraubenkopfs und das Gewinde der Schraube haben eine unterschiedliche Steigung, wodurch ein Heranziehen der Platte an den Knochen resultiert. Bei größeren Abständen zum Knochen kann durch Großfragmentschrauben die Platte an den Knochen herangezogen werden. Diese Schrauben werden später durch winkelstabile Schrauben ersetzt.

Den Fixateur-interne-Systemen ist zu eigen, dass die Schrauben in einem freien Winkel von bis zu über $30^{\circ}$ eingebracht werden können. Zudem können beide Platten

Tabelle 1

Vancouver-Klassiffkation nach

Masri u. Duncen [16]

Typ A:Trochanterfrakturen

Typ B: Frakturen im Prothesenbereich

- B1 bei fester Prothese

- $\mathrm{B} 2$ bei gelockerter Prothese und gutem Knochen

- B3 bei gelockerter Prothese und schlechtem Knochen

Typ C: Frakturen unterhalb der Prothese 


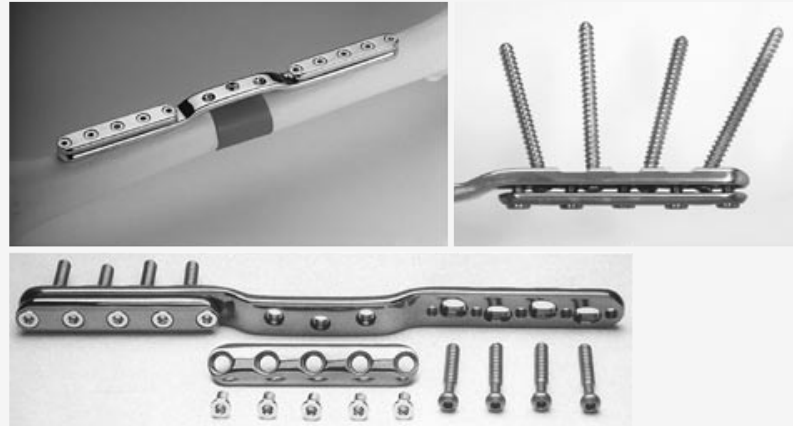

Abb. $1 \Delta$ Druckplattenfixateur interne: die Winkelstabilität wird durch Aufschrauben der Deckelplatte erreicht
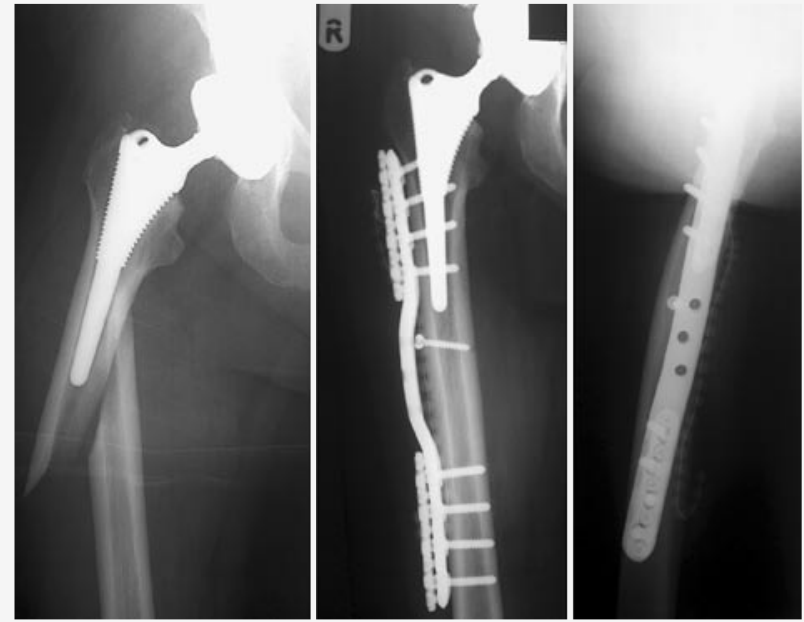

Abb. 3 Periprothetische Fraktur Typ B1 bei stabiler TEP, Versorgung mit Druckplattenfixateur
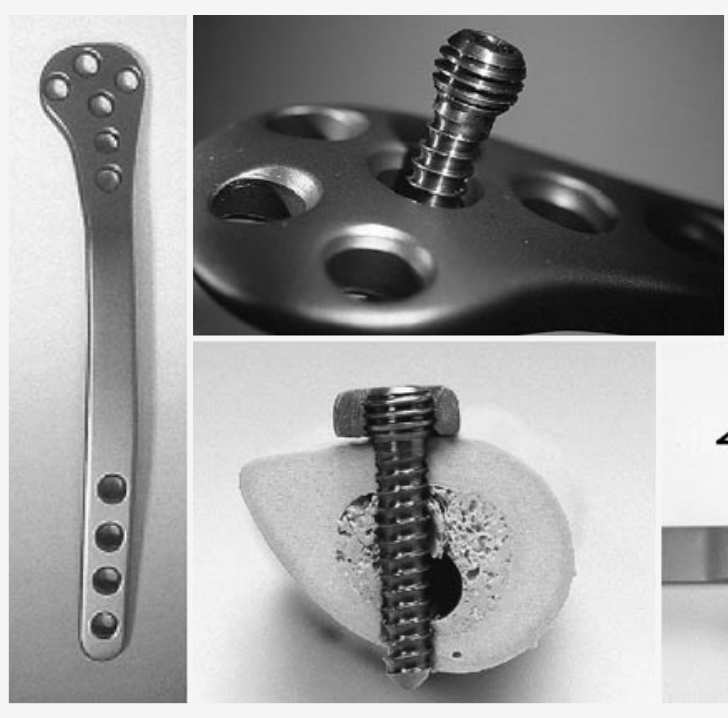

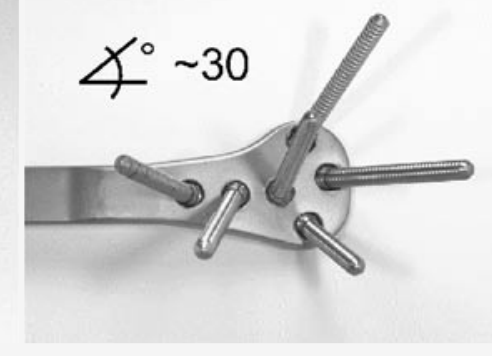

Abb. $2<$ KondylenTifix: als Links- und Rechtsversion in 4 Längen vorhanden mit herkömmlichen Schränkeisen gebogen und dem Knochen angepasst werden. Eine Verformung der Plattenlöcher durch den Biegevorgang ist unbedeutend, da diese Verformung beim Eindrehen von Gewindeformer und Schraube wieder aufgeweitet wird. Es resultiert eine hohe Variabilität, um auch an liegenden Prothesenimplantaten Schrauben platzieren zu können ( $\bullet$ Abb. 1, 2).

Auch und gerade bei osteoporotischem Knochen kann durch die Winkelstabilität eine hohe Stabilität erreicht werden. Die Schrauben-Plattenverbindung ist jederzeit wieder lösbar, sodass eine Materialentfernung ohne Einschränkung erfolgen kann.

Es existieren zahlreiche Klassifikationen für die periprothetischen Frakturen. Eine sehr einfache aber hilfreiche Klassifikation ist die Vancouver-Klassifikation nach Masri u. Duncen (• Tabelle 1 ).

\section{Patienten}

Wir berichten über unsere Ergebnisse bei periprothetischen Frakturen bei fester Prothese, also dem Typ B1 der VancouverKlassifikation. Die gelockerte Prothese erfordert ein anderes Vorgehen.

Von 1997 bis 2003 haben wir im Berufsgenossenschaftlichen Unfallkrankenhaus Hamburg 32 Patienten mit periprothetischen Frakturen operativ versorgt. Das Verhältnis weiblich/männlich betrug $21 \mathrm{zu} 11$. Das Durchschnittsalter lag bei 74,9 Jahre (56-87 Jahre). 18-mal handelte es sich um Frakturen bei einliegender Hüfttotalendoprothese und 13-mal bei einliegender Knieendoprothese. 1-mal lag sowohl eine Hüftals auch Knieprothese ein.

Der Druckplattenfixateur interne wurde 16-mal bei Hüfttotalendoprothese
(- Abb. 3), 2-mal bei Knietotalendoprothese und 1-mal bei Hüft- und Knietotalendoprothese eingesetzt. Der Kondylenfixateur interne wurde 2-mal bei liegender Hüfttotalendoprothese und 11-mal bei Knieendoprothese eingesetzt. 4-mal konnte ein minimal-invasiver Zugang verwendet werden (• Abb. 4a-c). In 4 Fällen wurde eine Verbundosteosynthese vorgenommen, in 7 Fällen eine Spongiosaplastik. In 1o Fällen wurden zur Infektionsprophylaxe PMMA-Ketten eingelegt.

\section{Ergebnisse}

Bei allen Patienten kam es zu einer primären Wundheilung ohne Infektion. 28 von 32 Patienten erreichten die Vollbelastung, teilweise unter Zuhilfenahme von Gehhilfen. Diese Gehhilfen wurden zum 


\section{Zusammenfassung $\cdot$ Abstract}

Teil aus frakturunabhängigen Gründen benötigt. 3 Patienten waren bereits vor der Fraktur bettlägerig. In 31 Fällen war die sofortige frühfunktionelle Behandlung möglich.

Es traten 7 Komplikationen auf. Eine Patientin verstarb an ihrer internistischen Begleiterkrankung im kardiopulmonalen Versagen. Es waren 4 postoperative Beinvenenthrombosen $\mathrm{zu}$ verzeichnen. 2-mal kam es zu proximalen Ausrissen der Fixateur-interne-Systeme bei liegender Knieendoprothese (• Tabelle 2). 1-mal handelte es sich um einen Ausriss eines Druckplattenfixateurs und 1-mal um einen Ausriss des Kondylenfixateurs. Beide Komplikationen konnten durch eine erneute Osteosynthese mittels Druckplattenfixateur beherrscht werden. Auch diese 2 Patienten erreichten nach der Revision die Vollbelastung.

\section{Diskussion}

Periprothetische Frakturen sind selten. In einer Serien von 30.000 Hüftendoprothesen, die in einem einzigen Institut eingesetzt wurden, fand sich eine postoperative Inzidenz von Femurfrakturen von 1,1\% nach der primären Hüftendoprothetik und von $4 \%$ nach Revisionseingriffen [5]. Durch höheres Lebensalter, steigende Implantationszahlen von Endoprothesen und vermehrte sportliche Aktivität werden periprothetische Frakturen in ihrer Häufigkeit zunehmen. Masri berichtet, dass postoperative periprothetische Frakturen in über $80 \%$ durch Bagatelltraumen entstehen [16]. Berry findet bei 50\% der Frakturen überhaupt kein Trauma in der Anamnese [5]. Als Risikofaktoren werden Osteoporose, Osteolysen, rheumatoide Arthritis, Kortikoidtherapie, zementierte Prothesen und Prothesenwechsel gesehen $[17,22,30]$.

\section{Stadiengerechte Therapie}

Die periprothetische Fraktur stellt nicht nur für den Chirurgen, sondern auch für das Implantat eine Herausforderung dar. Entscheidend für die Art der operativen Versorgung sind die Lokalisation der Fraktur in Bezug zur Prothese, die Knochenqualität und die Implantatstabilität. Diese Kriterien liegen der Vancouver-Klassifika-

Trauma Berufskrankh $2005 \cdot 7[$ Suppl 1]:S33-S38

DOI 10.1007/s10039-004-0941-2

c) Springer Medizin Verlag 2004

M. Faschingbauer · O. Pingen · C. Jürgens · D. Wolter

\section{Erfahrungen mit winkelstabilen Plattensystemen bei periprothetischen Frakturen}

\section{Zusammenfassung}

Multidirektionale Fixateur-interne-Systeme (Druckplattenfixateur, TiFix) ermöglichen beim polymorbiden älteren Patienten auch bei der periprothetischen Fraktur eine kurze und blutungsarme Operation. Bei der häufig vorliegenden schlechten Knochenqualität kann durch die Winkelstabilität der Platten eine hohe Festigkeit erreicht werden, sodass immer eine Übungsstabilität mit der Möglichkeit der frühfunktionellen Behandlung möglich ist. Durch die Op- tion, die Schrauben variabel einbringen zu können und die Platte dem Knochen anzumodellieren, kann selbst bei großvolumigen Endoprothesenimplantaten eine sichere Verankerung der Schrauben erreicht werden.

\section{Schlüsselwörter}

Periprothetische Fraktur · Femurfraktur . Winkelstabile Fixationssysteme.

Vancouver-Klassifikation · Endoprothesen

\section{Periprosthetic fractures - Experiences with angular stable plate osteosynthesis}

\begin{abstract}
In our opinion implants with multidirectional blocked screws are the ideal treatment for periprosthetic fractures even in the elderly and critically ill patient. The operation is not time-consuming and the blood loss is relatively low. In osteoporotic bone those fixateur intern systems with multidirectional screws give a high stability so that we rapidly achieve joint motion. With the possibility of fixing screws in various angles and
\end{abstract}

the possibility of contouring the plate to the bone we even achieve a rigid fixation in the presence of a voluminous prostheses.

\author{
Keywords \\ Periprosthetic fractures . \\ Vancouver classification - Femur fracture . \\ Internal fixator systems . \\ Angular stable condylar plate
}




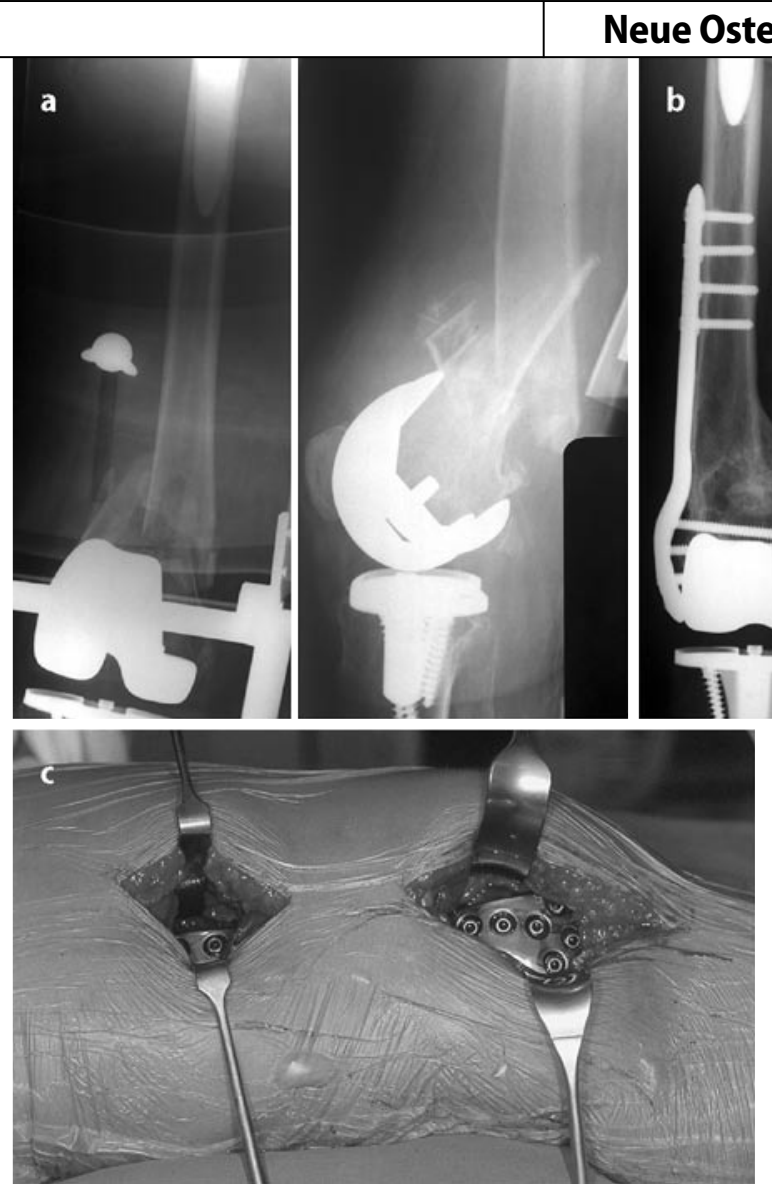

Abb.4a-c $<$ Periprothetische

Fraktur. a Frakturbilder bei liegendem Oberflächenersatz. b Versorgung mit KondylenTifix. c Minimal-invasives Vorgehen mit Einschieben der Platte

tion nach Duncen u. Masri zugrunde [8]. Als Basis dient die Frakturlokalisation am proximalen Femur. Sie wird in 3 Zonen eingeteilt.

Entsprechend dieser Klassifikation lässt sich ein stadiengerechtes Therapieschema ableiten.

Die Typ-A-Frakturen (Trochanterfrakturen) werden in der Regel konservativ behandelt, nur bei größerer Dislokation des Trochanter ist ein operatives Vorgehen nötig.

Die Frakturen vom Typ B2 und $B_{3}$ mit Prothesenlockerung werden in der Regel mit einer Revisionsprothese versorgt, wobei sowohl die zementierte als auch unzementierte Version, Verriegelungsprothesen und die Anlagerung von autologen oder allogenen Spongiosa- bzw. Knochenchips oder „allograft struts“ diskutiert werden $[4,9,19,26,28,29]$.

Frakturen vom Typ $C$ werden wie einfache distale Femurfrakturen behandelt.

Bei Frakturen vom Typ B1 also bei ungelockerter Prothese sollte eine konservative Therapie auf Grund der schlechten Ergebnisse die Ausnahme sein [14]. Standard ist das operative Vorgehen. Dabei kommen
Platten in Kombination mit Schrauben und Zerklagen, Spezialplatten, Titanbänder in Kombination mit Knochenchips und winkelstabile Implantate zum Einsatz. Von verschiedenen Autoren werden aber auch Revisionsprothesen bei B1-Frakturen eingesetzt, obwohl die einliegende Prothese nicht gelockert ist [23].

\section{Plattenosteosynthese-Techniken}

AO-Standardplatten und Schrauben werden bei B1-Frakturen eingesetzt. Die konventionelle Plattenosteosynthese zeigt jedoch eine hohe Komplikationsrate $[3,22]$. So berichtet Aigner über 30-50\% Versagensraten [2]. Parks empfiehlt, Schrauben an der Prothesenspitze zu vermeiden [18]. Deshalb wurden Spezialplatten entwickelt, die mit Zerklagen oder Bändern kombiniert werden.

Die Ogden-Platte (Fa. Zimmer) war eine der ersten Platten, die eine Kombination von Platte mit Kabel oder Schraube ermöglichte. Zenni berichtet über 19 Patienten, die er mit einer Ogden-Platte versorgte. Es waren 2 verzögerte Knochenbruchheilungen, 1 Pseudarthrose, 1 Platten- bruch, 1 Plattenausriss und 1 Fraktur unterhalb der Platte zu verzeichnen. Die OgdenPlatte ist nicht für periprothetische Frakturen bei liegenden Knieendoprothesen geeignet [32].

Das Dall-Miles-Kabel-Plattensystem (Fa. Howmedica) zeigt ähnliche Ergebnisse. Bei dieser Platte besteht die Möglichkeit, sowohl distal als auch proximal eine Fixierung mit Zerklagen vorzunehmen. Venu beschreibt 3 Pseudarthrosen bei 12 Patienten, die mit einer Dall-MilesPlatte versorgt wurden [27]. Tandross erzielte mit der Dall-Miles-Platte nur bei 3 von 7 periprothetischen Frakturen einen knöchernen Durchbau [25].

Die Mennen-Platte erwies sich als zu schwach. Ahuja beschreibt 75\% Komplikationen mit der Mennen-Platte [1]. Kamineni berichtet über 5 Versager bei 5 Patienten und erachtet die Mennen-Platte als nicht geeignet für den Einsatz bei periprothetischen Frakturen [15]. Bei der MennenPlatte handelt es sich um eine Platte mit Krallen, die das Femur umfassen. Die Fixierung erfolgt durch das Umbiegen dieser Krallen und nicht durch Schrauben.

Aus der Revisionschirurgie bei Schaftlockerungen ist die Verwendung von kortikalen Allografts, so genannten Strut Grafts (Strut = englisch Stütze/Strebe) bekannt. Chandler berichtete 1993 über 19 periprothetische Frakturen, die er mit Allograft Strut stabilisierte. Bei 16 von diesen 19 Patienten kam es zu einem knöchernen Durchbau innerhalb von 4 1/2 Monaten. In einer Kombination von einer Metallplatte auf der lateralen Kortikalis und einem Allograft Strut auf der gegenüberliegenden Kortikalisseite beschreibt Chandler einen klinischen Erfolg bei 21 von $22 \mathrm{~Pa}$ tienten mit periprothetischen Frakturen $[6,7]$. Auch Aigener berichtet bei einem Vergleich von allogenen Struts mit der konventionellen Plattenosteosynthese von einem gutem bis sehr guten Ergebnis bei 7 von 7 Patienten [2].

\section{Winkelstabile Systeme}

Nachdem sich winkelstabile Fixateur-interne-Systeme vor allem bei Problemfrakturen bewährt hatten, berichteten wir 2001 über unsere Ergebnisse mit dem Kondylen-Tifix und dem Druckplattenfixateur bei periprothetischen Frakturen [10, 11, 12, 
13]. Wolter u. Seide konnten nachweisen, dass winkelstabile Fixateur-interne-Systeme (Fa. Litos) vor allem auch bei osteoporotischem Knochen einen deutlich verbesserten Halt gegenüber nicht winkelstabilen Systemen zeigten [21, 31]. Diese Fixateur-interne-Systeme erlauben es, die Schrauben variabel mit einem Winkel von

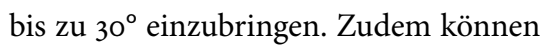
die Platten den anatomischen Gegebenheiten angepasst und gebogen werden.

Bei 31 der 32 von uns mit winkelstabilen Fixateur-interne-Systemen versorgten Patienten konnte eine sofortige frühfunktionelle Behandlung erreicht werden. Ein Patient verstarb frakturunabhängig. $28 \mathrm{~Pa}$ tienten erreichten die Vollbelastung, $3 \mathrm{~Pa}$ tienten waren bereits vor der Fraktur bettlägerig.

2-mal kam es zu proximalen Ausrissen der Fixateur-interne-Systeme bei liegenden Knieprothesen. Hier handelte es sich um technische Fehler. Die Gewinde im proximalen Femur wurden mit einem zu kleinen Gewindeschneider angelegt, sodass es zu einem medialen Ausbruch eines Knochenfragments beim Einbringen der Schrauben kam. Dies führte letztendlich zum Ausriss des Fixateurs. Durch Verwenden eines längeren Fixateurs wurde die Problematik überwunden.

In unserer Serie traten erfreulicherweise die sonst häufig beschriebenen Probleme der Infektion und der Pseudarthrosebildung nicht auf. Als Ursachen sehen wir die hohe Stabilität und Verminderung der Mikrobewegungen bei der Winkelstabilität sowie die Verwendung von Refobacinund Palacos-Ketten.

\section{Vergleich mit dynamischer Kondylenschraube}

Suprakondyläre Femurfrakturen bei liegenden Knieprothesen werden mittels DCS, retrograden Nägeln oder winkelstabilen Fixateur-interne-Systemen stabilisiert. Retrograde Nägel sind nur bei einem Oberflächenersatz möglich, der vom Design eine Nagelung zulässt $[24,30]$. Andere winkelstabile Fixateursysteme wie z. B. das LISS-System lassen weder das Anmodellieren der Platte noch ein variables Einbringen der Schrauben zu.

Wenn wir das Kondylen-Tifix-System mit der dynamischen Kondylenschraube

Tabelle 2

In der Studie aufgetretene Komplikationen

\begin{tabular}{|lllll} 
& $\begin{array}{l}\text { Postoperativ } \\
\text { verstorben }\end{array}$ & $\begin{array}{l}\text { Tiefe } \\
\text { Beinvenenthrombose }\end{array}$ & Plattenausriss & $\mathbf{n = 7}$ \\
Allgemein & 1 & 4 & 2 & 5 \\
\hline Lokal & & & 2 \\
\hline
\end{tabular}

(DCS) vergleichen, findet sich ein geringeres Volumen der winkelstabilen Schrauben bei deutlich größerer knöcherner Abstützfläche im Knochen. Vergleicht man die knöcherne Abstützfläche und das Volumen von 4 winkelstabilen Schrauben einer Fixateur-interne-Platte mit einer DCS mit zusätzlicher Spongiosaschraube, welche regelhaft nötig ist, so zeigt sich, dass das Volumen der DCS samt Spongiosaschraube ca. $38 \%$ größer ist. Die Auflagefläche von 4 winkelstabilen TiFix-Schrauben dagegen ist um ca. 70\% größer, als die einer DCS mit Spongiosaschraube. Dieses bedeutet für die Fixateur-interne-Platte eine größere knöcherne Abstützung bei geringerer Knochenschädigung durch großvoluminöse Implantate [11].

\section{Bewertung der Ergebnisse}

Die Komplikationsrate bei periprothetischen Frakturen wird in der Literatur mit ca. 35\% angegeben [20]. Somit sind unsere Ergebnisse mit 2 Versagensfällen der Montage sowie 5 allgemeinen Komplikationen bei 32 Patienten als günstig anzusehen.

Die flächenhafte Lasteinleitung, die Schonung des häufig geschwächten Knochens, seine höhere Belastbarkeit, die geringere Mikrobewegung und das leichtere operative Einbringen sind Gründe für diese Ergebnisse.

\section{Korrespondierender Autor Dr. M. Faschingbauer}

Berufsgenossenschaftliches Unfallkrankenhaus, Bergedorfer Straße 10, 21033 Hamburg E-Mail: M.Faschingbauer@buk-hamburg-de

Interessenkonflikt: Keine Angaben

\section{Literatur}

1. Ahuja S, Chatterji S (2002) The Mennen femoral plate for fixation of periprosthetic femoral fractures following hip arthroplasty. Injury 33: 47-50
2. Aigner C, Marschall C, Reischl N, Windhagen R (2002) Kortikale Strut Grafts, eine Alternative zur konventionellen Plattenosteosynthese bei periprothetischer Fraktur. Z Orthop Ihre Grenzgeb 140: 328-333

3. Beals RK, Tower SS (1996) Periprosthetic fractures of the Femur. Clin Orthop 327: 238-246

4. Berry DJ (2002) Management of periprosthetic fractures: the hip. Arthroplasty 17 [Suppl 1]: 11-13

5. Berry DJ (1999) Epidemiology: hip and knee. Orthop Clin North Am 30: 183-190

6. Chandler HP, King D, Limbird R et al. (1993) The use of cortical allograft struts for fixation of fractures associated with well-fixed total joint prostheses. Semin Arthroplasty 4: 99-107

7. Dennis MG, Simon JA, Kummer FJ, Koval KJ, Di Cesare PE (2001) Fixation of periprosthetic femoral shaft fractures: a biomechanical comparsion of two techniques. J Orthop Trauma 15: 177-180

8. Duncan CP, Masri BA (1995) Fractures of the femur after hip replacement. Instr Course Lect 44: 293304

9. Eingartner C, Volkmann R, Winter E, Stoll B, Weise K, Weller S (2003) Die Marknagelung periprothetischer Femurfrakturen mittels Verriegelungsprothese. Akt Traumatol 33: 267-271

10. Faschingbauer M, Stütz A, Wenzl M (2001) Fraktur- und Pseudarthrosenversorgung im Oberschenkelbereich durch Titan-Fixateur-interne-Systeme mit frei wählbarer Schraubenlage. Trauma Berufskrankh 3 [Suppl 4]: S434-S438

11. Faschingbauer $M$, Wolter $D$, Stütz $A$, Reimers $N$ (1999) Distale Oberschenkelmehrfragmentfraktur. Ideale Indikation für ein winkelstabiles Implantat? Trauma Berufskrankh 1: 337-343

12. Faschingbauer $M$, Pingen $O$, Strametz $S$, Wenzl ME, Jürgens C (2003) Rekonstruktion und Stabilisierung nach fehlverheilten Frakturen und Pseudarthrosen im Femurbereich mit winkelstabilen Implantaten. Trauma Berufskrankh 5 [Suppl 1]: S62S66

13. Faschingbauer $M$, Krüss $C$, Wenzl $M E$, Wolter $D$ (2001) Die Versorgung periprothetischer Schaftfrakturen im Oberschenkelbereich mit Fixateur interne Systemen. Hefte Unfallchirurg 283: 367-368

14. Kamineni S, Vindlacheruvu R, Ware HE (1999) Periprosthetic femoral shaft fractures treated with plate and cable fixation. Injury 30: 261-268

15. Kamineni S, Ware HE (1999) The Mennen plate: unsuitable for the elderly femoral periprosthetic fractures. Injury 30: 257-260

16. Masri BA, Meek RM, Duncan CP (2004) Periprosthetic fractures evaluation and treatment. Clin Orthop 420: 80-95

17. Nelson CL (2002) Periprosthetic fractures of the femur following hip arthroplasty. Am J Orthop 31: 221-223

18. Park MS, Lee YK, Yang KH, Shin SJ (2003) Management of periprosthetic femoral fractures. J Arthroplasty 18: 903-906 


\section{Leserservice: Themenübersicht}

19. Scholz R, Pretzsch M, Matzen P, von Salis-Soglio GF (2003) Zur Behandlung periprothetischer Femurfrakturen bei Hüftendoprothesen. Z Orthop 141: 296-302

20. Schwarz E, ScharfW (2003) Der Andocknagel - eine neue Möglichkeit zur Behandlung der periprothetischen Oberschenkelfraktur bei liegender Endoprothese der Hüfte. Akt Traumatol 33: 284-291

21. Seide K, Zierold W, Wolter D, Kortmann HR (1990) Einfluß einer winkelstabilen Platten-SchraubenVerbindung und unterschiedlicher Schraubendicken auf die Statik der Plattenosteosynthese, eine FEM-Studie. Unfallchirurg 93: 552-558

22. Siegmeth $A$, Menth-Chiari W, Wozasek GE, Vecsei V (1998) Die periprothetische Femurschaftfraktur. Unfallchirurg 101: 901-906

23. Spinger B, Daniel J, Berry, MD, Lewallen DG (2003) Treatment of periprosthetic femoral fractures following total hip arthroplasty with femoral component revision. J Bone Joint Surg Am 85: 2156-2162

24. Spitaler R, Reichetseder J, Rappold G, Leixnering $M$ Hertz H (2003) Periprothetische Fraktur bei ipsilateraler Hüft- bzw. Knieendoprothese: Das Akutversorgungskonzept - ein operativer Algorithmus. Akt Traumatol 33: 272-280

25. Tandross TS, Nanu AM, Buchanan MJ et al. (2000) Dall-Miles plating for periprosthetic B 1 fractures. J Arthroplasty 15: 47-51

26. Tsiridis E, Haddad FS, Gie GA (2003) The management of periprosthetic femoral fractures around hip replacements. Injury 34: 95-105

27. Venu KM, Koka R, Garikipati R, Shenaca Y, Madhu TS (2001) Dall-miles cable and plate fixation for the treatment of periprothetic femoral fractures-analysis of results in 13 cases. Injury 32: 395-400

28. Wagner H (1987) Revisionsprothese für das Hüftgelenk bei schwerem Knochenverlust. Orthopäde 16: 295-300

29. Wahl B, Graßhoff H, Meinecke I, Neumann HW (2001) Ergebnisse der operativen Therapie periprothetischer Femurschaftfrakturen bei Hüfttotalendoprothesen. Zentralbl Chir 126: 50-54

30. Wick M, Müller E J, Muhr G (2001) Suprakondyläre Femurfrakturen bei Knieendoprothesen. Unfallchirurg 104: 410-413

31. Wolter $D$ (1991) Der Plattenfixateur interne für lange Röhrenknochen. In: Wolter D, Zimmer W (Hrsg) Die Plattenosteosynthese und ihre Konkurrenzverfahren. Springer, Berlin Heidelberg New York

32. Zenni EJ, Pomeroy DL, Caudle RJ (1988) Ogden plate and other fixations for fractures complicating femoral endoprostheses. Clin Orthop 231: 83-90

33. http://www.bqs-online.delVeröffentlichunglQualitätsreport2002
„Trauma und Berufskrankheit“ bietet aktuelle Beiträge zu Fragen der Unfall- und Wiederherstellungschirurgie, der Berufserkrankungen sowie zu Themen der Unfallverhütung, der operativen und konservativen Therapie.

Möchten Sie ein bereits erschienenes Heft nachbestellen? Die folgenden Ausgaben können Sie direkt bei unserem Kundenservice zum Preis von je EUR 35,- beziehen:

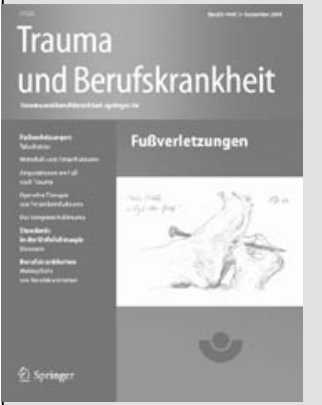

\section{Heft 3/2004}

Leitthema:

- Fußverletzungen

Standards in der Unfallchirurgie:

- Unterarmr

Berufskrankheit:

- Meldepflicht von Berufskrankheiten

Heft 4/2004

Leitthema:

- Resorbierbare Materialien

Standards in der Unfallchirurgie:

- Ellengelenk, Oberarm

Originalien:

- Knorpelzelltransplantation

\section{Heft 1/2005}

Leitthema:

- Frakturen im Wachstumsalter

Standards in der Unfallchirurgie:

- AC-Gelenkverletzungen

Berufskrankheit:

- Rückenkolleg für Krankenschwestern

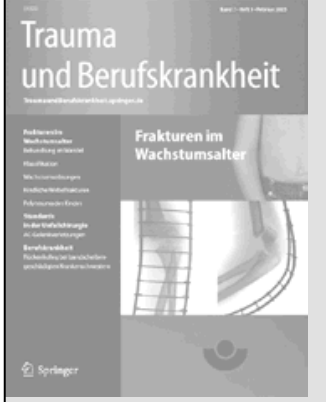

\section{Vorschau Heft 2/2005}

Leitthema:

- Intramedulläre Kraftträger

Standards in der Unfallchirurgie:

- Hand

BG-Heilverfahren:

- Heilverfahren bei Sprunggelenksfrakturen

- Einbindung von Psychotherapeuten

So erreichen Sie unseren Kundenservice:

Springer-Verlag

Kundenservice Zeitschriften

Haberstr. 7, 69126 Heidelberg

Tel.: +49 6221 345-4303

Fax: $+496221345-4229$

E-Mail:SDC-journals@springer-sbm.com

\section{TraumaundBerufskrankheit.springer.de}

